\title{
Subclinical persisting pulmonary hypertension in chronic neonatal lung disease
}

\author{
Dominic Fitzgerald, Nick Evans, Peter Van Asperen, David Henderson-Smart
}

\begin{abstract}
The development of pulmonary hypertension is one of the adverse factors in the outcome of infants with chronic neonatal lung disease (CNLD). The purpose of this cross sectional study was to evaluate the prevalence and degree of pulmonary hypertension in a cohort of survivors of CNLD stable in air. Pulmonary artery pressure was assessed using its inverse correlation with the ratio of time to peak velocity and right ventricular ejection time (TPV:RVET) as measured from Doppler velocity time signals in the main pulmonary artery. A normal ratio is $\geqslant 0 \cdot 35$, a possibly low ratio lies between 0.31 and 0.35 , and a definitely low ratio is $<0 \cdot 31$. The subjects were divided into three groups. Group A comprised 58 infants with oxygen dependence and an abnormal chest radiograph at 28 days of age; group $B$ comprised 18 infants with oxygen dependence and a normal chest radiograph at 28 days of age; and group $C$ (controls) comprised 21 siblings without oxygen dependence by 10 days and a normal chest radiograph.
\end{abstract}

There were significant differences in mean (SD) TPV:RVET ratio between group A $0.346(0.045)$, group B 0.335 $(0.057)$, and groups A+B $0.344(0.048)$ when compared with group $C$ controls $0.385(0.034)$. The prevalence of a definitely low TPV:RVET ratio suggesting a raised pulmonary artery pressure was $19 \%$ in group $A, 39 \%$ in group $B, 24 \%$ in groups $\mathbf{A}+\mathbf{B}$, and none in group $\mathbf{C}$. There were no clinical signs of pulmonary hypertension in any patient studied. Stepwise multiple linear regression failed to find significant associations with antenatal or neonatal putative risk factors. Additionally, there were no associations with childhood respiratory morbidity. These data suggest a high prevalence of subclinical pulmonary hypertension in CNLD patients. It is speculated that occult hypoxaemia may be occurring in this group of infants.

(Arch Dis Child 1994; 70: F118-F122)

Chronic neonatal lung disease (CNLD) is an ongoing cause of significant morbidity and mortality for graduates of neonatal intensive care units despite the advent of surfactant treatment and advances in technology. ${ }^{1}$ In severe CNLD, pulmonary hypertension is seen as a serious and frequent complication. ${ }^{2}$
Pulmonary hypertension has been documented in preterm infants in the acute phase of the respiratory distress syndrome utilising invasive and non-invasive means. ${ }^{3-6}$ More recently, Evans and Archer showed that about one third of a group of preterm infants recovering from hyaline membrane disease still had Doppler echocardiographic evidence of raised pulmonary artery pressure when discharged home in air. ${ }^{4}$ The main factor which distinguished these infants from those in whom pulmonary artery pressure returned to normal was the length of time spent in oxygen. This raises the likelihood that there is a spectrum of raised pulmonary artery pressure in CNLD and that not only severe cases are affected.

The natural history of pulmonary hypertension after hospital discharge in infants with CNLD who are stable in air has not to our knowledge been previously examined. Therefore, the aim of this study was to examine the prevalence and degree of pulmonary hypertension in a cross sectional cohort of such infants using a validated Doppler echocardiographic method. ${ }^{3-5} 78$ Furthermore, we aimed to examine whether any perinatal or postneonatal factors were associated with the presence of pulmonary hypertension.

\section{Patients and methods}

A total of 97 patients were assessed. There were 76 patients with CNLD and 21 sibling controls. The 76 study patients were derived from a total of 186 patients with CNLD managed at King George V Hospital from 1985 to 1990. CNLD was defined as either oxygen dependence at 28 days with an abnormal chest radiograph $(n=141$, group A) or oxygen dependence at 28 days and a normal chest radiograph $(n=45)$, group B). A letter explaining the study and a questionnaire was sent to the parents of 148 patients whose current address could be found. Thirteen were returned as address unknown. Thus there were 135 potential patients for this study. Fourteen patients were excluded from the study: two had moved overseas, four had moved interstate, five patients refused echocardiographs or other aspects of the study, three had structural heart disease (one atrial septal defect; two mild pulmonary valve stenosis). Consequently, there remained 121 patients eligible to study. Of that number, 77 volunteered to participate in the study. One patient was uncooperative on the day he was examined and refused to undergo echocardiography. Therefore 76 out of a possible 121 patients $(63 \%)$ participated in the study. 
The control patients (group C) were siblings of study patients $(n=21)$. There were 12 preterm infants (including two 26 week triplets and a 29 week triplet) and nine term infants. Four of the preterm controls required intubation (maximum period was two days) and one required nasal continuous positive airways pressure for five days. All preterm controls were stable in air by 10 days of age. None of the term controls $(n=9)$ required ventilation or supplemental oxygen.

All patients were studied once between February and October 1992. The patients were participating in a wider prospective study of clinical outcome of CNLD patients managed at King George V Hospital from 1985 to 1990 . This study consisted of a self administered questionnaire that was corroborated at the time of consultation, a clinical examination, a chest radiograph, echocardiography, skin prick testing to common inhaled and ingested allergens as well as pulmonary function testing including a histamine challenge in those children over 5 years of age. In this paper we report the echocardiographic results, some questionnaire details, and physical findings. The questionnaire included basic demographic details about the family: 11 questions about the pregnancy, labour, and delivery; 13 questions about wheeze and asthma; 10 questions about medical conditions manifesting after infancy; three questions about medical and community follow up; six questions about family and environmental history; and seven questions about psychosocial issues pertaining to caring for a child with chronic illness. Other aspects of this study will be reported separately.

All echocardiographic studies were performed by the same operator (NE) utilising an Accuson 128XP/10 scanner incorporating pulse wave and colour flow Doppler. A $5 \mathrm{MHz}$ transducer was used for all studies. The echocardiographer was blinded to the perinatal history and physical findings on examination of each patient studied. The pulmonary artery was imaged from the parasternal long axis view. The pulsed Doppler range gate was placed in the centre of the main pulmonary artery distal to the pulmonary valve. The echocardiogram was recorded onto videotape and measurements were taken at a later date using the Accuson's incorporated measurement callipers. Only laminar, non-turbulent flow from the centre of the artery was used for measurement. The time to peak velocity (TPV) was defined as the time interval between the Doppler velocity time signal

Table 1 Comparison of demographic data for all patients within study groups; figures are mean (range)

\begin{tabular}{llll}
\hline & $\begin{array}{l}\text { Birth weight } \\
(g)\end{array}$ & $\begin{array}{l}\text { Gestational } \\
\text { age (weeks) }\end{array}$ & $\begin{array}{l}\text { Age at study } \\
\text { (months) }\end{array}$ \\
\hline $\begin{array}{l}\text { Group A: CLD and oxygen dependent }(\mathrm{n}=58) \\
\text { Group B: oxygen dependent }(\mathrm{n}=18)\end{array}$ & $1035(654-3010)$ & $27(24-35)$ & $48(12-90)$ \\
$\begin{array}{l}\text { Groups A and B: CNLD }(\mathrm{n}=76) \\
\text { Group C: preterm and term sibling controls }\end{array}$ & $1265(745-4350)$ & $28(25-41)$ & $45(17-84)$ \\
$\quad$ combined $(\mathrm{n}=21)$ & $2600(860-4700)$ & $34(25-41)$ & $62(13-144)$ \\
$\quad \begin{array}{l}\text { Subgroup C1: preterm controls }(\mathrm{n}=12) \\
\text { Subgroup C2: term controls }(\mathrm{n}=9)\end{array}$ & $1876(915-3400)$ & $31(26-36)$ & $45(13-84)$ \\
& $3566(2395-4700)$ & $39(38-41)$ & $87(47-144)$ \\
\hline
\end{tabular}

$\mathrm{CLD}=$ chronic lung disease. leaving the baseline and reaching its peak velocity. The right ventricular ejection time (RVET) was the time between the signal leaving the baseline and returning to the baseline. The TPV:RVET ratio was averaged from a mean of 5 cardiac cycles (range 3-10). For each echocardiogram the R-R interval was also measured. A normal TPV:RVET ratio taken from the data of Kosturakis et al was 0.35 or greater. ${ }^{8}$ Cases of known structural heart disease $(n=3)$ were excluded from the study. No sedation was used for any patient studied. All patients had been stable in air for at least nine months.

Results are reported as mean (SD) or range. The measure of statistical significance among the mean of two or more groups was performed with one way analysis of variance. Correlations between TPV:RVET ratios and antenatal or neonatal putative risk factors were assessed utilising multiple linear regression analysis. Similarly, stepwise linear regression was used to assess association between markers of childhood respiratory morbidity. Linear regression was used to compare TPV:RVET ratios with $R-R$ intervals. All statistical analysis was performed with a standard statistical software package (SPSS, Microsoft Corporation, 1990). A p value less than 0.05 was considered significant.

Approval for the study was obtained from the ethics review committees of both King George V Hospital For Mothers and Babies and The Children's Hospital, Camperdown. Written informed parental consent was obtained prior to the commencement of the study.

\section{Results}

The birth weight, gestational age, and age at the time of assessment for the three groups $(A+B+C)$ is outlined in table 1 . Group $C$ had a significantly higher mean birth weight and gestational age than the other groups and the term controls were studied at a significantly older age than other groups $(p<0.05)$. None of the other differences were significant.

The TPV:RVET ratios for the groups are illustrated in fig 1 . The mean (SD) TPV:RVET ratio for group A was 0.346 $(0.045)$, group B was $0.335(0.057)$, and groups A+B was $0.344(0.048)$. All of these were significantly lower than the mean for group C controls, $0.385(0.034) ; \mathrm{p}<0.005$.

The TPV:RVET ratio is an indirect method of assessing pulmonary artery pressure, all such methods are subject to a degree of error. In light of this it was believed inappropriate to label infants with TPV:RVET ratio just below 0.35 as definitely abnormal. This is emphasised by the two preterm control infants with TPV:RVET ratios just below the normal range. Because of this, we have arbitrarily divided those ratios below 0.35 based on the lowest TPV:RVET ratio in the control group into possibly low $(0 \cdot 31-0.34)$ and definitely low $(<0 \cdot 31)$.

The prevalence of a low TPV:RVET ratio $(<0.35)$ in each of these groups was as follows: 


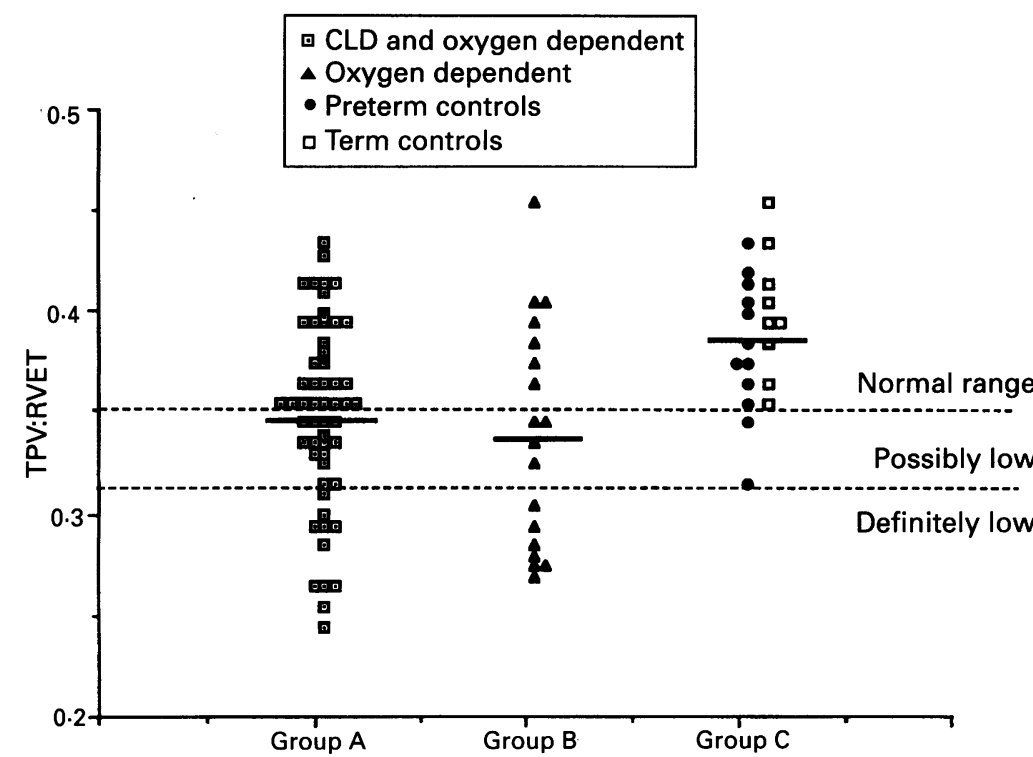

Figure 1 TPV:RVET ratios for each study group divided into normal, possibly low, and definitely low ranges. The mean ratio for each group is marked by the horizontal black line. Group $C$ control means are different from group $A$ and group $B(p<0.005)$. Groups $A$ and $B$ means are not significantly different. $C L D=$ chronic lung disease.

group A $43 \%$ with $19 \%$ of the whole group definitely low, group B $61 \%$ with $39 \%$ definitely low, and groups A+B $47 \%$ with $24 \%$ definitely low. The control groups $\mathrm{C}$ had two infants $(9 \%)$ in the possibly low range.

Group C was subsequently divided into preterm (group C1) and term (group C2) controls. Group $\mathrm{C} 1$ comprised 12 subjects with two infants $(17 \%)$ having a possibly low TPV:RVET ratio. These two preterm controls had TPV:RVET ratios of 0.31 and 0.34 and were two of 26 week gestation triplets. The third triplet had CNLD (group A) and had a TPV:RVET ratio in the normal range $(0 \cdot 36)$. For the preterm controls with a normal ratio the mean (SD) TPV:RVET ratio was $0.388(0.025)$ which was almost identical with the term control mean of $0.391(0.035)$. All nine subjects in group C2 had normal TPV:RVET ratios.

The TPV:RVET ratio was plotted against corrected postnatal age up to 36 months and

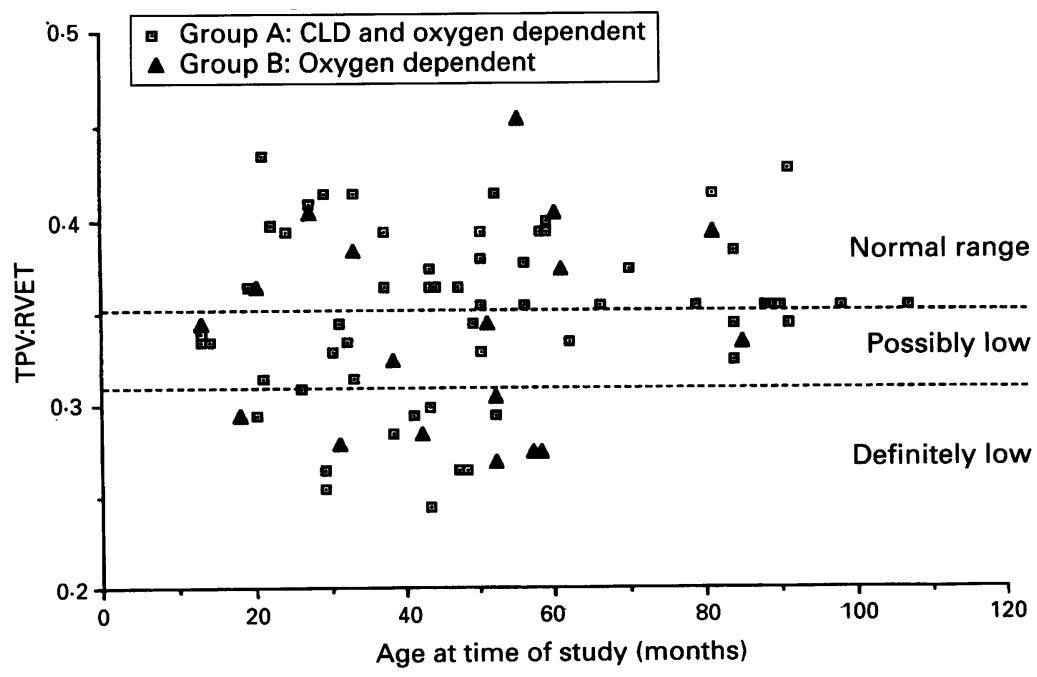

Figure $2 T P V: R V E T$ ratio plotted age at the time of study for each infant in groups $A$ and $B$ divided into normal, possibly low, and definitely low ranges. $C L D=$ chronic lung disease. chronological age after this time. This is represented in fig 2 for groups A and B. There was a trend toward higher TPV:RVET ratio with age. No infant had a TPV:RVET ratio $<0.32$ beyond 5 years of age.

In order to assess the effect of heart rate on the TPV:RVET ratio the $R-R$ interval was used. For all 96 study patients, there was no statistically significant correlation between TPV:RVET ratio and R-R interval ( $r=0.004)$. Similarly, there was no significant correlation between the R-R interval and TPV:RVET ratio for all 21 controls $(r=0.234)$ or for all patients with TPV:RVET ratios in the normal range $(r=-0.064)$. Consequently, TPV:RVET ratio was not corrected for heart rate in this analysis.

In assessing the neonatal putative risk factors, no statistically significant associations were found. Associations were sought for the following antenatal risk factors: primiparity, two or more antenatal visits, treated infections in pregnancy, method of conception, and cigarette smoking. Perinatal risk factors included: gender, birth weight, centile, gestational age, maternal age at confinement, prolonged rupture of membranes, threatened premature labour, antenatal maternal steroid cover, treated symptomatic persistent patent ductus arteriosus, maximum peak inspiratory pressure, number of days ventilated, and number of days in supplemental oxygen. Of these, only birth weight approached statistical significance $(p=0.058)$. In table 2 the profiles of subjects with CNLD (groups $A+B$ ) with normal, possibly low, and definitely low TPV:RVET ratios are contrasted. There were no significant differences in the gestational age, birth weight, nor days in oxygen.

Respiratory morbidity in younger children was also addressed and again there were no statistically significant associations between raised pulmonary artery pressure and the prevalence of bronchiolitis, current wheezing, asthma, croup, number of intercurrent upper respiratory tract infections per year, attendance at day care, parental smoking at home, maternal asthma, and maternal atopy.

There were no statistically significant associations between raised pulmonary artery pressure and systolic or diastolic blood pressure centiles, weight centiles, or the presence of chest wall deformity at the time of study. None of the children studied had clinical signs of pulmonary hypertension.

\section{Discussion}

This study has demonstrated that a significant number of survivors of CNLD continue to demonstrate raised pulmonary artery pressures well into early childhood. This was studied using the non-invasive measurement of the TPV:RVET ratio. There exists sufficient correlation between the TPV:RVET ratio and measured pulmonary artery pressure at catheterisation to validate this as an indirect method for assessing pulmonary artery pressure, ${ }^{7910}$ regardless of whether the raised pressure is due to increased pulmonary blood 
Table 2 Comparison of parameters in 76 study infants with normal, possibly low, and definitely low TPV:RVET ratio; figures are mean (SD)

\begin{tabular}{lccc}
\hline & $\begin{array}{c}\text { Normal ratio } \\
\left(\begin{array}{l}0.35) \\
(n=40)\end{array}\right.\end{array}$ & $\begin{array}{l}\text { Possibly low ratio } \\
(<0.35 \text { and }>0.31) \\
(n=18)\end{array}$ & $\begin{array}{l}\text { Definitely low } \\
\text { ratio }(<0.31) \\
(n=18)\end{array}$ \\
\hline TPV:RVET ratio & $0.380(0 \cdot 026)$ & $0.330(0.01)$ & $0 \cdot 276(0.018)$ \\
Days in oxygen & $64(28)$ & $71(35)$ & $65(50)$ \\
Gestational age (weeks) & $27(1)$ & $27(2)$ & $28(3)$ \\
Birth weight (g) & $1066(380)$ & $985(217)$ & $1195(812)$ \\
Age at time of study (months) & $53(24)$ & $43(27)$ & $40(10)$
\end{tabular}

Apart from the TPV:RVET, none of the differences are statistically significant.

flow or primary lung pathology. Based upon the work of Kosturakis et $a l,{ }^{8}$ who defined normal values for the TPV:RVET ratio in 15 paediatric subjects, a TPV:RVET ratio $<0.35$ would approximate to a mean pulmonary artery pressure of $20 \mathrm{~mm} \mathrm{Hg}$. Similarly, a TPV:RVET ratio of 0.25 would approximate to a mean pulmonary artery pressure of $50 \mathrm{~mm}$ $\mathrm{Hg}$. The alternative non-invasive method of measuring pulmonary hypertension relies on Doppler echocardiographic measurement of the peak velocity of a tricuspid incompetence jet. However, this method precludes measuring pulmonary artery pressure in those patients without tricuspid incompetence who have milder elevations of pulmonary artery pressure. Clearly, the latter method would not have suited this study which reviewed pulmonary artery pressure across the spectrum of CNLD. The TPV:RVET ratio, while marginally less accurate in approximating pulmonary artery pressure than the tricuspid incompetence method, ${ }^{11}$ can be measured in all infants.

The confounding variables for interpretation of the TPV:RVET ratio are heart rate, Doppler sample position for measuring the ratio, right ventricular dysfunction, and the presence of large left to right shunts. ${ }^{91012}$ As the TPV:RVET ratio may fall with increasing heart rate we plotted the $R-R$ interval against the TPV:RVET ratio. There was no statistically significant association between these variables. Consequently, we did not analyse the TPV:RVET ratio corrected for heart rate. The constant measurement site is detailed in the methods section and there was no subjective evidence of right ventricular dysfunction nor any left to right shunting in any patients studied.

These data support the belief that raised pulmonary artery pressure is not limited to those infants with severe CNLD. This is further illustrated by the spectrum of raised pulmonary artery pressure demonstrated across the range of severity of CNLD children in early childhood. While not knowing their respective pulmonary artery pressures in infancy, it seems unlikely that children who have survived CNLD would have raised pulmonary artery pressure for other reasons. There is a trend toward the normal range with increasing age for the TPV:RVET ratio in both groups $A$ and $B$. This improvement in TPV:RVET ratios. with age may reflect lung healing and alveolar growth in early childhood. Similarly, the range of TPV:RVET ratios for group C controls was similar to the previously published normal range. ${ }^{8}$ Specifically, 19 of our 21 controls had TPV:RVET values within this normal range. All term infants had normal TPV:RVET ratios. The two low values $(0.34$ and 0.31 ) were in two of three surviving 26 week triplets. Could a low TPV:RVET ratio be a feature of prematurity rather than a manifestation of CNLD? This seems unlikely in that two thirds of the infants studied by Evans and Archer had TPV:RVET ratios which returned to the normal range prior to discharge from hospital. ${ }^{4}$ A TPV:RVET ratio remaining low in Evans and Archer's study was strongly associated with prolonged oxygen dependency.

Our study has identified a large subgroup (24\%) of very low birthweight CNLD infants (only two of our 76 CNLD infants had birth weights over $1500 \mathrm{~g}$ ) with raised pulmonary artery pressure as defined by a definitely low TPV:RVET ratio that are not readily distinguished from their CNLD contemporaries with normal pulmonary artery pressure. Furthermore, there was a further $24 \%$ of study patients with possibly low TPV:RVET ratios who may have raised pulmonary artery pressure. This latter group represents the 'grey zone' between normal and definitely abnormal and reflects the continuum of severity that exists in many manifestations of CNLD which tend to confound arbitrary definitions so often applied in this condition.

There were no significant associated postneonatal intensive care factors that were apparent by questionnaire, history, or physical examination in our study. We would speculate that this subclinical raised pulmonary artery pressure may result from hypoxaemia adversely affecting reactive pulmonary vasculature during the early weeks and months of life. That subclinical hypoxaemia occurs is well documented, ${ }^{13-15}$ although the extent of this problem may be underestimated. It is possible that the desire to avoid the serious complication of retinopathy of prematurity in combination with suboptimal oxygen monitoring (for example by spot oximetry alone) may leave a significant number of CNLD patients at risk of borderline hypoxaemia which may be sufficient to aggravate a predisposition to pulmonary hypertension. Clearly, the prevalence of raised pulmonary artery pressure among a group of infants in whom much time, expertise, and financial resources have been invested is of concern.

That such a large proportion have echocardiographic but not clinical evidence of pulmonary hypertension in early childhood may reflect the subtle nature of this problem and the need for more aggressive surveillance of CNLD infants in order to corroborate these findings. Continuous recordings of oximetry, especially during periods of sleep and feeds, should be considered in all CNLD patients before discharge from the nursery as a simple, noninvasive baseline parameter of respiratory status. Should excessive periods of oxygen desaturation occur, then the scope for more detailed testing with polysomnography and echocardiography may be indicated. Failing that, further time in supplemental oxygen until the oximetry remains satisfactory should be encouraged. 
There remains considerable controversy with regards to the appropriate definition of CNLD. All the infants in this study were oxygen dependent at 28 days. They were divided into two groups depending on the presence (group A) or absence (group B) of radiological abnormalities. The lack of difference in TPV:RVET ratios between these two groups would suggest that the incorporation of radiological criteria into the definition is inappropriate and that the pathophysiology in the two groups is the same. The data was reanalysed using the CNLD definition of Shennan et al ${ }^{16}$ of oxygen dependency at 36 weeks' postconceptional age in those with birth weight $<1500 \mathrm{~g}$ (only two of our 76 CNLD patients had birth weights greater than $1500 \mathrm{~g}$ ). This reduced the number of CNLD patients by $55 \%$ but only marginally increased the prevalence of a low TPV:RVET ratio to $48 \%$. CNLD is almost certainly a disease process which represents a continuous spectrum of neonatally sustained lung damage and to apply dichotomous definitions seems inappropriate. There is a spectrum of disturbance of lung function which extends into early childhood. ${ }^{14} 15$ 17-19 Similarly, these data have shown that the spectrum of raised pulmonary artery pressure which has been observed in the neonatal period ${ }^{520}$ continues into early childhood in survivors of CNLD.

This was a cross sectional study and as such the results need to be interpreted with caution. However, we would hypothesise that the presence of a low TPV:RVET ratio may identify a group of infants who need particularly close oxygen monitoring and respiratory follow up. To this end we have embarked upon a prospective longitudinal study of CNLD infants commencing in the neonatal period to define the natural history of this subclinical pulmonary hypertension.

1 Parker RA, Lindstrom DP, Cotton RB. Improved survival accounts for most but not all of the increase in bronchoaccounts for most but not all of the increase in

2 Goodman G, Perkin RM, Anas NG, Sperling DR, Hicks DA, Rowen M. Pulmonary hypertension in infants with bronchopulmonary dysplasia. F Pediatr 1988; 112: 67-72.
3 Walther FJ, Benders MJ, Leighton JO. Persistent pulmonary hypertension in premature neonates with severe respiratory distress syndrome. Pediatrics 1992; 90: 899-904.

4 Evans NJ, Archer LNJ. Doppler assessment of pulmonary artery pressure during recovery from hyaline membrane disease. Arch Dis Child 1991; 66: 802-4.

5 Evans NJ, Archer LNJ. Doppler assessment of pulmonary artery pressure and extrapulmonary shunting in the acute phase of hyaline membrane disease. Arch Dis Child 1991; 66: 6-11.

6 Stahlman M, Blankenship WJ, Shepart FM, Gray J, Young WC, Malam AF. Circulatory studies in clinical hyaline membrane disease. Biol Neonate 1972; 20: 300-20.

7 Kitabatake A, Inoue M, Asao M, et al. Non-invasive evaluation of pulmonary hypertension by a pulsed Dopple technique. Circulation 1983; 68: 302-9.

8 Kosturakis D, Goldberg SJ,. Allen HD, Loebor C. Doppler echocardiographic prediction of pulmonary arterial hypertension in congenital heart disease. Am $\mathcal{F}$ Cardiol 1984; 53: $1110-5$.

9 Serwer GA, Cougle AG, Eckerd JM, Armstrong BE. Factors affecting use of the doppler determined time from flow onset to maximal pulmonary artery velocity for measurement of pulmonary artery pressure in children. Am f Cardiol 1986; 58: 352-6.

10 Matsuda M, Sekiguehi T, Sugishita Y, Kuwaka K, Iida K, Ito I. Reliability of non-invasive estimates of pulmonary hypertension by pulsed Doppler echocardiography. Br Heart f 1986; 56: 158-64.

11 Chan KL, Currie PJ, Seward JB, Hagler DJ, Mair DD, Tajik AJ. Comparison of three Doppler ultrasound methods in the prediction of pulmonary artery pressure. 7 Am Coll Cardiol 1987; 9: 549-54.

12 Panidis IP, Ross J, Mintz GS. Effect of sampling site on assessment of pulmonary artery blood flow by Doppler echocardiography. Am 7 Cardiol 1986; 58: 1145-7.

13 O'Brodovich HM, Mellins RB. Bronchopulmonary dysplasia unresolved neonatal acute lung injury. Am Rev Respir Dis 1985; 32: 694-709.

14 Sekar KC, Duke JC. Sleep apnea and hypoxemia in recently weaned premature infants with and withou bronchopulmonary dysplasia. Pediatr Pulmonol 1991;10 $112-6$.

15 Singer L, Martin RJ, Hawkins SW, Benson-Szekely LJ, Yamashita TS, Waldemer AC. Oxygen desaturation complicates feeding in infants with bronchopulmonary dysplasia after discharge. Pediatrics 1992; 90: 380-4.

16 Shennan AT, Dunn MS, Ohlsson A, Lennox K, Hoskin EM. Abnormal pulmonary outcomes in premature EM. Abnormal pulmonary outcomes in premature infants: prediction from oxygen requirem

17 Blayney M, Kerem E, Whyte H. O'Brodovich $H$. Bronchopulmonary dysplasia: improvement in lung function between 7 and 10 years of age. $\mathcal{F}$ Pediatr 1991; 118: $201-6$.

18 Mallory JB Jnr, Chaney H, Mutich RL, Motoyamma EK Longitudinal changes in lung function during the first three years of premature infants with moderate to severe bronchopulmonary dysplasia. Pediatr Pulmonol 1991; 11: 8-14.

19 Hakulinen $\mathrm{AL}$, Heinonen $\mathrm{K}$, Lansimies $\mathrm{E}$, Kiekara $\mathrm{O}$ Pulmonary function and respiratory morbidity in school age children born prematurely and ventilated for neonatal age children born prematurely and ventilated for neonatal respiratory

$20 \mathrm{Gill} \mathrm{AB}$, Weindling AM. Pulmonary artery pressure changes in the very low birthweight infant developing chronic lung disease. Arch Dis Child 1993; 68: 303-7. 